\title{
Steady State In A Markov Process
}

Franklin Lowenthal, (E-mail: flowenth@ csuhayward), California State University, Hayward Massoud Malek, California State University, Hayward

\begin{abstract}
It is well known that a Markov process whose transition matrix is regular approaches a steadystate distribution, or equilibrium distribution. To find these steady-state probabilities requires the solution of a system of linear homogenous equations. However, the matrix of this system is singular and thus the system has infinitely many solutions. This obstacle is overcome by replacing one of the equations of the linear homogenous system by the linear non-homogeneous equation that simply expresses the requirement that the steady-state probabilities sum to one. But which equation of the original system should be chosen to be the one replaced. This brief article demonstrates that any of the equations of the original linear system can be selected as the one to be replaced; no matter which one is selected for replacement; the revised linear system will have the same unique solution.
\end{abstract}

\section{INTRODUCTION}

$\mathrm{n}$ all that follows, we will denote by $\theta$, the zero column vector, by $\mathrm{e}_{\mathrm{k}}$ the k-th column of the identity matrix I, and by e, the column vector $(1,1, \ldots, 1)^{\mathrm{t}}$. A vector with positive components is said to be positive. The set of all eigenvalues of an $\mathrm{n} \times \mathrm{n}$ matrix $\mathrm{A}$ is called the spectrum of $\mathrm{A}$ and is denoted by $\sigma$ (A). A maximal eigenvalue is an eigenvalue with the largest modulus; the corresponding eigenvector is called a maximal eigenvector. A non-negative $\mathrm{n} \times \mathrm{n}$ matrix A, where the sum of the entries of each row adds to one (i.e., $\mathrm{Ae}=\mathrm{e}$ ) is called stochastic; this vector e is a maximal eigenvector and one is a maximal eigenvalue of $\mathrm{A}$ (see [5]). The norm of a vector $\mathrm{u}=$ $\left(\mathrm{u}_{1}, \mathrm{u}_{2}, \ldots, \mathrm{u}_{\mathrm{n}}\right)^{\mathrm{t}}$ is defined as follows: $\quad\|\mathrm{u}\|=\sum_{k=1}^{n}\left|\mathrm{u}_{\mathrm{k}}\right|$.

It is well known that a Markov process whose transition matrix $\mathrm{P}$ is regular (i.e., some power of $\mathrm{P}$ will consist entirely of positive entries) approaches a steady-state distribution, or equilibrium distribution. The steady-state probabilities are of special interest in many business applications as they can be viewed as the market shares of competing firms after the passage of a long period of time. To find these steady-state probabilities requires the solution of a system of linear homogenous equations. However, the matrix of this system is singular and thus the system has infinitely many solutions. Various texts on operations research and quantitative business methods (see for example [1, pp. 677-681], [2, pp. 265-271], [8, 571-575], [10, p.979] and [11, p. 692]) including the most advanced texts such as [7, pp. 547-548], the popular mathematical book for Management Science [9, pp. 182-186], and the highly respected book by Hillier and Liberman [4, pp. 381-382], without any proof or justification, simply state that in order to obtain the steady-state vector for $\mathrm{P}$, one of the equations in the linear homogeneous system $\left(\mathrm{I}-\mathrm{P}^{\mathrm{t}}\right) \mathrm{v}=\theta$ should be replaced by the requirement $\mathrm{e}^{\mathrm{t}} \mathrm{v}=1$. But this prescription leaves two questions unanswered. First, does it matter which equation we choose to delete? No. In fact, the negative of the sum of any subset of $n-1$ equations from this system equals the remaining equation. Second, is the solution of the revised linear non-homogeneous system always unique? We will give a concise proof based on the Perron-Frobenius theorem, that the linear nonhomogeneous system will have a unique steady-state solution.

We will present the problem through the vehicle of an example from marketing. 


\section{EXAMPLE}

Suppose the consumer has a choice of purchasing one of five competing brands of a product; call the brands $\mathrm{A}, \mathrm{B}, \mathrm{C}, \mathrm{D}$ and $\mathrm{E}$ respectively. Assume purchases are made once a week, with the decision on which brand to purchase depending only on the brand that was purchased in the previous week. A Markov chain can describe the process; we assume the transition matrix $\mathrm{P}$ is given by the $5 \times 5$ matrix below.

$\mathrm{P}=\quad \begin{array}{lllll}0.4 & 0.3 & 0.2 & 0.1 & 0.0 \\ 0.5 & 0.2 & 0.1 & 0.1 & 0.1 \\ 0.0 & 0.4 & 0.4 & 0.1 & 0.1 \\ 0.2 & 0.2 & 0.2 & 0.2 & 0.2 \\ 0.3 & 0.1 & 0.1 & 0.1 & 0.4\end{array}$

Note that $\mathrm{P}$ is stochastic. The entry in the $\mathrm{i}$-th row and $\mathrm{j}$-th column represents the probability that a consumer who last purchased brand $i$ will switch to brand $j$. The transition matrix $P$ is regular. Thus it is possible in some number of periods to go from any state $i$ to any state $j$. The marketing analyst is interested in whether after a period of time, this process will approach an equilibrium state in which each brand ends up with a steady share of the market.

If we raise the matrix $\mathrm{P}$ of our example to the 8 th power we obtain the matrix:

$\mathrm{P}^{8}=\quad \begin{array}{lllll}0.311112 & 0.260344 & 0.203192 & 0.111111 & 0.114240 \\ 0.311114 & 0.260343 & 0.203191 & 0.111111 & 0.114241 \\ 0.311107 & 0.260284 & 0.203153 & 0.111111 & 0.114344 \\ 0.311109 & 0.260283 & 0.203152 & 0.111111 & 0.114346 \\ 0.311111 & 0.260280 & 0.203150 & 0.111111 & 0.114348\end{array}$

Note that the 5 rows of this matrix are almost identical. If instead we raised $\mathrm{P}$ to the 16 th power we would obtain:

$\mathrm{P}^{16}=\begin{array}{llllll}0.311111 & 0.260317 & 0.203175 & 0.111111 & 0.114286 \\ 0.311111 & 0.260317 & 0.203175 & 0.111111 & 0.114286 \\ 0.311111 & 0.260317 & 0.203175 & 0.111111 & 0.114286 \\ 0.311111 & 0.260317 & 0.203175 & 0.111111 & 0.114286 \\ 0.311111 & 0.260317 & 0.203175 & 0.111111 & 0.114286\end{array}$

These rows are identical for at least the first 6 significant digits. This phenomenon constitutes the content of a wellknown theorem for regular transition matrices (see Kemeny-Snell [6] for example).

\section{RESULTS}

In all that follows, $\mathrm{P}$ represents an $\mathrm{n} \times \mathrm{n}$ regular stochastic transition matrix. The Perron-Frobenius theorem (see [3, p. 53]) states that under the assumptions that $P$ is regular (the term primitive is also used), both $P$ and $P^{t}$ have a maximal eigenvalue which is unique, algebraically simple, and positive; also each matrix has a maximal positive eigenvector. Since P is stochastic, e is a maximal eigenvector corresponding to the maximal eigenvalue one; we denote by $\pi$, the normalized positive maximal eigenvector of $\mathrm{P}^{\mathrm{t}}$; so $\mathrm{e}^{\mathrm{t}} \pi=1$. The facts that $\sigma\left(\mathrm{P}^{\mathrm{N}}\right)=\left\{\lambda^{\mathrm{N}}\right.$; $\lambda \in \sigma(\mathrm{P})\}$ and the unique maximal eigenvalue $\lambda_{0}=1$ of $\mathrm{P}$ is simple, imply that $\mathrm{P}^{\mathrm{N}}$ converges to a matrix $\mathrm{Q}$ of rank one as $\mathrm{N} \rightarrow \infty$. Since e and $\pi$ are also maximal eigenvectors of the idempotent matrices $\mathrm{Q}$ and $\mathrm{Q}^{\mathrm{t}}$, respectively, we conclude that $\mathrm{Q}=\mathrm{e} \pi^{\mathrm{t}}$. Thus $\pi^{\mathrm{t}}$, any common row of the limiting matrix $\mathrm{Q}$ is the steady-state or equilibrium probabilities vector for $\mathrm{P}$ (see also [6, p. 70]). Clearly, finding a positive solution of the equation $\left(\mathrm{I}-\mathrm{P}^{\mathrm{t}}\right) \mathrm{v}=\theta$ and then normalizing the resulting vector is a faster and more accurate method than taking $\mathrm{P}$ to a large power.

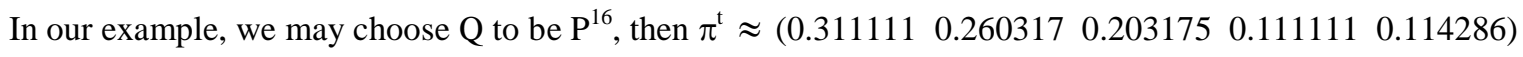
will be the steady-state probabilities vector satisfying the following conditions: (I-P $\left.\mathrm{P}^{\mathrm{t}}\right) \pi \approx \theta$ and et $\pi \approx 1$. 
Choose any $\mathrm{k}=1,2 \ldots, \mathrm{n}$ and form the $\mathrm{n} \times \mathrm{n}$ matrix $\mathrm{P}_{\mathrm{k}}$ by deleting the $\mathrm{k}$-th row of the matrix $\mathrm{I}-\mathrm{P}^{\mathrm{t}}$ and inserting a final row consisting of the row vector $e^{t}$. Note that $P_{k} \pi^{t}=e_{n}$; we will answer our question on the uniqueness of the solution $\mathrm{P}_{\mathrm{k}} \mathrm{V}=\mathrm{e}_{\mathrm{n}}$, but first we need to prove the following lemma.

Lemma. Any set of n-1 rows of the matrix I-P $\mathrm{P}^{\mathrm{t}}$ is linearly independent.

Proof: Suppose the contrary, then there exists another eigenvector $\mathrm{u}(\mathrm{I}-\mathrm{P}) \mathrm{u}=\theta$ or $\mathrm{u}^{\mathrm{t}}\left(\mathrm{I}-\mathrm{P}^{\mathrm{t}}\right)=\theta^{\mathrm{t}}$ with at least one zero component, as we can take a linear combination of those $\mathrm{n}-1$ rows plus zero times the remaining row to get $\mathrm{u}^{\mathrm{t}}$. Since $\{\mathrm{e}, \mathrm{u}\}$ must be linearly independent, the geometric multiplicity of the maximal eigenvalue is at least two, a contradiction.

Theorem. The following conditions hold for $\mathrm{P}_{\mathrm{k}}$ :

(i) $\quad P_{k}$ is invertible.

(ii) $\pi$ is a unique solution of the linear non-homogeneous system: $P_{k} v=e \_n$.

(iii) $\pi$ is the last column of the matrix $\mathrm{P}_{\mathrm{k}}^{-1}$.

Proof: Suppose $P_{k}$ is singular, by the lemma, the first $n-1$ rows of $P_{k}$ are linearly independent; thus the row vector $e^{t}$ must be a linear combination of the first n-1 rows of $P_{k}$. Since the product of every one of the first $n-1$ rows of $P_{k}$ and $\pi$ is zero, we have $\mathrm{e}^{\mathrm{t}} \pi^{\mathrm{t}}=0$, a contradiction with $\mathrm{e}^{\mathrm{t}} \pi^{\mathrm{t}}=1$. Hence $(i)$ follows.

Since $\mathrm{P}_{\mathrm{k}} \pi=\mathrm{e}_{\mathrm{n}}$ and $\mathrm{P}_{\mathrm{k}}$ is invertible, (ii) follows.

Finally, part (iii) is obtained from $\mathrm{P}_{\mathrm{k}} \mathrm{P}_{\mathrm{k}}^{-1}=\mathrm{I}$.

Although solving the system $\mathrm{P}_{\mathrm{k}} \mathrm{V}=\mathrm{e}_{\mathrm{n}}$ is a more efficient mean of finding the steady-state vector than using the inverse of $\mathrm{P}_{\mathrm{k}}$. the latter method is widely used in Operations Research. In fact, the popular mathematical book for Management Science [9] finds $\pi$ only through the matrix $\mathrm{P}_{\mathrm{k}}^{-1}$.

We will illustrate the resulting algorithm with our example using the inverse of $\mathrm{P}_{3}$.

$\mathrm{P}_{3}=\begin{array}{lllll}0.6 & -0.5 & 0.0 & -0.2 & -0.3 \\ -0.3 & 0.8 & -0.4 & -0.2 & -0.3 \\ -0.1 & -0.1 & -0.1 & 0.8 & -0.1 \\ 0.0 & -0.1 & -0.1 & -0.2 & 0.6 \\ 1.0 & 1.0 & 1.0 & 1.0 & 1.0\end{array}$

The inverse of this matrix is found to be

$\begin{array}{llllll} & 1.5 & 0.625 & 0.236111 & 0.375 & 0.311111 \\ \mathrm{P}_{3}^{-1}= & -0.07143 & 0.803571 & -0.23611 & -0.375 & 0.260317 \\ -1.21429 & -1.33929 & -1.23611 & -1.375 & 0.203175 \\ 0.00 & 0.00 & 1.111111 & 0.00 & 0.111111 \\ & -0.21429 & -0.08929 & 0.125 & 1.375 & 0.114286\end{array}$

The entries in the last column of the matrix $\mathrm{P}_{3}^{-1}$ are the desired steady-state probabilities which are the same as the entries in any common row vector of $\mathrm{P}_{3}{ }^{16}$. If we had deleted a different row, the inverse would be different but the last column would be the same.

Remark. Defining $\mathrm{P}_{\mathrm{k}}$ involves relabeling some of the rows of the matrix I-Pt; to avoid this, we may define the invertible matrix $S_{k}$ by replacing the k-th row of I-P ${ }^{t}$ with the vector $e^{t}$ then $\pi^{t \text { will }}$ be unique solution of the system $P_{k} e=$ $\mathrm{e}_{\mathrm{k}}$ or the k-th column of the matrix $\mathrm{S}_{\mathrm{k}}^{-1}$. 


\section{CONCLUSION}

Our results show that any of the two following simple algorithms can be implemented on any spreadsheet software to find the steady-state or equilibrium probabilities for any regular Markov process. The first algorithm is the traditional approach used in operations research; it is based on finding the inverse of a matrix. The second, a more efficient algorithm uses a linear homogeneous system of only n-1 equations and the matrix P-I which is faster to compute than I-P.

If $\mathrm{P}$ denotes the transition matrix of a regular Markov process, then:

\section{Algorithm 1.}

Step 1. Write down the matrix I-P.

Step 2. Compute the transpose of the matrix in Step 1.

Step 3. Form a matrix $P_{k}$ by removing any "undesirable" row of the matrix in Step 2 and insert a final row consisting of all ones.

Step 4. Find the inverse of the matrix $\mathrm{P}_{\mathrm{k}}$.

Step 5. The steady-state probabilities are the entries in the last column of $\mathrm{P}_{\mathrm{k}}^{-1}$

\section{Algorithm 2.}

Step 1. Write down the matrix P-I.

Step 2. Form a matrix R by removing any "undesirable" column of the matrix in Step 1.

Step 3. Find a positive solution $\mathrm{v}_{0}$ of the linear homogeneous system $\mathrm{v}^{\mathrm{t}} \mathrm{R}=\theta^{\mathrm{t}}$.

Step 4. The steady-state probabilities vector $\pi^{\mathrm{t}}=\mathrm{v}_{0}{ }^{\mathrm{t}} /\left\|\mathrm{v} \_0\right\|$.

\section{REFERENCES}

1. Budnick, Frank S., Mc Leavey, Dennis, and Mojena, Richard, Principles of Operations Research for Management, 2nd Edition, IRWIN, 1988.

2. Buffa, Elwood S. and Dyer, James, Management Science/Operations Research, Model Foundation and Solution Methods. John Wiley \& Sons, 1977.

3. F. R. Gantmacher, Matrix Theory, Vol. 2, Chelsa, New York, 1959.

4. Hillies, Frederick S. and Liberman, Gerald J., Introduction to Operations Research, 3rd Edition, HoldenDay, 1980.

5. A. S. Householder, The Theory of Matrices in Numerical Analysis, Dover Publications, New York, 1964.

6. Kemeny, John and Snell, J. Laurie, Finite Markov Chains, D. Van Nostrand Co. Inc., 1960.

7. Markland, Robert E., Topics in Management Science, 3rd Edition, Wiley, 1989.

8. Render, Barry and Stair, Ralph, Quantative Analysis For Management, Allyn and Bacon, Inc. 1982.

9. Schmidt, J. William, Mathematical Foundations for Management Science and Systems Analysis, Academic Press, 1974.

10. Taha, Hamdy A., Operations Research, an Introduction, Mc Millan, 1992.

11. Winston, Wayne L., Operations Research, Applications and Algorithm, $3^{\mathrm{RD}}$ Edition, Duxbury Press, 1994 